\title{
Antifungal susceptibility testing for yeasts: how, when and why, according to the new EUCAST guidelines
}

\author{
Esther Manso ${ }^{1,8}$, Claudio Farina ${ }^{2,8}$, Stefano Andreoni ${ }^{3,8}$, Marco Conte ${ }^{4,8}$, Paolo Fazii ${ }^{5,8}$, \\ Gianluigi Lombardi ${ }^{6,8}$, Silvana Sanna ${ }^{7,8}$ \\ I. Laboratorio di Analisi Chimico-Cliniche e Microbiologia, AOU Ospedali Riuniti di Ancona, Ancona \\ 2. UOC Microbiologia e Virologia, AO Ospedale San Carlo Borromeo, Milano \\ 3. Laboratorio di Microbiologia, AOU Maggiore della Carità, Novara \\ 4. Laboratorio di Microbiologia, AO Ospedale Domenico Cotugno, Napoli \\ 5. Laboratorio di Analisi Chimico-Cliniche e Microbiologia, Ospedale Santo Spirito, Pescara \\ 6. SC Microbiologia e Virologia, AO Ospedale Niguarda-Ca' Granda, Milano \\ 7. Istituto di Microbiologia, Università degli Studi di Sassari, Sassari \\ 8. Comitato di Studio per I a Micologia (CoSM), Associazione Microbiologi Clinici Italiani, Milano
}

Key words: Lievito, Antimicogramma, EUCAST

I test di sensibilità agli antifungini per i lieviti: come, quando e perché. Alla luce delle proposte EUCAST

\section{SUMMARY}

Antifungal susceptibility testing of yeasts and the development of interpretive breakpoints are nowadays very important topics both for the increasing incidence of invasive mycoses and number of available antifungal drugs and for the appearance of acquired resistance.

The Subcommittee on Antifungal Susceptibility Testing (AFST) of the European Committee on Antibiotic Susceptibility Testing (EUCAST) has recently released both the operative standards to evaluate the fermenting yeasts susceptibility against antimycotic drugs and the breakpoints for infections due to some Candida species and for some of the available antifungals.

Antifungal susceptibility testing is mandatory on a routine base for: I. therapeutic failure, 2. breakthrough fungemias, 3. on yeasts isolated from patients who recently received prophylactic antifungal treatments, and 4 . when the deep-seated infection is due to less common yeasts, with an unknown susceptibility pattern.

The Medical Mycology Committee (CoSM) of the Italian Society of Clinical Microbiologists (AMCLI) adopted the interpretive breakpoints of both CLSI and EUCAST. The operating procedure for antifungal susceptibility testing according to EUCAST is supported by the recommendation to use, when clinically necessary, validated commercial methods (Vitek2, Sensititre, E-test).

Finally, the CoSM reports the recommendations for a correct management of antifungal susceptibility testing.

\section{INTRODUZIONE}

I test di sensibilità dei miceti e lo sviluppo di breakpoint interpretativi sono argomenti di sempre crescente attualità, per l'incremento dell'incidenza delle micosi invasive, dell'aumento delle classi di molecole antifungine e dell'emergenza di resistenze acquisite.

I breakpoint clinici si basano sul presupposto che i ceppi fungini che si caratterizzano per valori di MIC a un antifungino superiore a determinate concentrazioni rispondono in modo significativamente inferiore a cicli di terapia con quel farmaco proprio per l'impossibilità di conseguire in vivo concentrazioni teoricamente terapeutiche.

Nel management di un paziente con infezione fungina invasiva la scelta dell'antimicotico da utilizzare in terapia si basa sostanzialmente sull'identificazione della specie fungina responsabile del quadro clinico. La resistenza acquisita agli antifungini è meno frequente in ambito micologico rispetto a quanto non si osservi con i batteri. Tuttavia, è stata osservata l'emergenza di resistenze in particolare là dove una carica elevata di miceti è esposta a terapia antifungina per lunghi periodi.

Ceppi fungini resistenti sono stati isolati in pazienti con candidosi orofaringea e con aspergilloma, specie se trattati per lunghi periodi con azoli. Al contrario, fenomeni di resistenza sono descritti più raramente nei pazienti con candidemia o con aspergillosi invasiva.

Il Subcommittee on Antifungal Susceptibility Testing (AFST) dell'European Committee on Antibiotic Susceptibility Testing (EUCAST) ha definito gli standard operativi per rilevare la sensibilità dei lieviti fermentanti e dei miceti filamen-

\section{Corresponding author: Claudio Farina}

UOC Microbiologia e Virologia, AO "Ospedale San Carlo Borromeo"

Via Pio II, 3 - 20153 Milano - Tel.: +39 240222456 - Fax: +39 240222829

E-mail: farina.claudio@sancarlo.mi.it 
tosi (11), ed ha proposto valori di breakpoint per le infezioni sostenute da alcune specie di Candida e per alcuni tra gli antifungini disponibili.

\section{L'evoluzione della standardizzazione dei test di sensibilità agli antifungini}

E ben noto che, per il successo di una terapia antifungina, sono più importanti i fattori dell'ospite che il risultato, in sé, dei test di sensibilità in vitro. Infatti, la sensibilità dei test in vitro non predice il successo della terapia, mentre la resistenza in vitro ne predice il fallimento terapeutico (test di resistenza). Sebbene i test di sensibilità in vitro siano abitualmente utilizzati per selezionare l'antifungino che si caratterizza per una maggiore probabilità di essere clinicamente attivo, è possibile che il ruolo principale di questi test sia la rilevazione di una resistenza. Questi concetti, di natura strettamente microbiologica, devono essere integrati con l'analisi delle variabili farmacodinamiche: solo l'integrazione delle due competenze, infatti, consente di interpretare in modo clinicamente utile i valori in vitro di MIC.

D'altra parte, è altrettanto ben noto come la Micologia Medica sia disciplina relativamente recente. Se si considera che tra la disponibilità della prima classe di molecole ad attività antibatterica (i sulfamidi, nel 1935) e l'amfotericina B, il primo antifungino ad essere utilizzato nel 1960, sono intercorsi venticinque anni, non può essere motivo di stupore il ritardo con cui venne pubblicata in un documento del National Clinical Committee for Laroratory Standard (NCCLS) statunitense, nel 1992, la prima proposta relativa ai criteri di standardizzazione dei test in vitro di chemiosensibilità agli antifungini nei confronti di alcuni lieviti (NCCLS Document M27-P) e poi dei miceti filamentosi (3).

La Tabella 1 riporta l'iter temporale della disponibilità di documenti NCCLS/CLSI in merito alla standardizzazione dell'antimicogramma.

Da allora tuttavia notevolissimo è stato l'impulso scientifico alla definizione di criteri operativi standardizzati per l'esecuzione e l'interpretazione

Tabella I. Cronistoria dei documenti NCCLS/CLSI di standardizzazione dei test di sensibilità agli antifungini

1982 NCCLS istituzione del Subcommittee on Antifungal Susceptibility Testing (NCCLS - Area Committee for Microbiology) 1985 NCCLS pubblicazione dell'NCCLS Document M20-CR, vol. 5, n. I7 “Antifungal Susceptibility Testing”'(Committee Report)

1992 NCCLS pubblicazione dell'NCCLS Document M27-P “Reference Method for Broth Dilution Antifungal Susceptibility Testing of Yeasts; Proposed Standard"

1997 NCCLS pubblicazione dell'NCCLS Document M27-A “Reference Method for Broth Dilution Antifungal Susceptibility Testing of Yeasts; Approved Standard"

1998 NCCLS pubblicazione dell'NCCLS Document M38-P "Reference Method for Broth Dilution Antifungal Susceptibility Testing of Conidium-Forming Filamentous Fungi; Proposed Standard"

2002 NCCLS pubblicazione dell'NCCLS Document M27-A2 "Reference Method for Broth Dilution Antifungal Susceptibility Testing of Yeasts; Approved Standard"

2002 NCCLS pubblicazione dell'NCCLS Document M38-A “Reference Method for Broth Dilution Antifungal Susceptibility Testing of Conidium-Forming Filamentous Fungi; Approved Standard"

2004 NCCLS pubblicazione dell'NCCLS Document M44-A “Method for antifungal disk diffusion susceptibility testing of yeast; Approved Guidelines"

2008 CLSI pubblicazione del CLSI M27-A3 "Reference Method for Broth Dilution Antifungal Susceptibility Testing of Yeasts; Approved Standard"

2008 CLSI pubblicazione del CLSI M27-S3 "Reference Method for broth dilution:Antifungal susceptibility testing of yeast; Third informational supplements"

2008 CLSI pubblicazione del CLSI Document M38-A2 "Reference Method for Broth Dilution Antifungal Susceptibility Testing of Conidium-Forming Filamentous Fungi; Approved Standard"

2009 CLSI pubblicazione del CLSI Document M44-A2 "Method for antifungal disk diffusion susceptibility testing of yeast; Guidelines"

2009 CLSI pubblicazione CLSI M44-S3 "Zone diameter interpretative standards, corresponding Minimal Inhibitory Concentration (MIC) interpretative breakpoints, and quality control limits for antifungal disk diffusion susceptibility testing of yeast; third Informational supplement"

2007 CLSI pubblicazione del CLSI M44-S2 “Zone diameter interpretative standards and corresponding Minimal Inhibitory Concentration (MIC) interpretative breakpoints, Informative supplement"

2009 CLSI pubblicazione del CLSI M5I-P "Method for antifungal disk diffusion susceptibility testing of nondermatophytes filamentous fungi;Approved Guideline"

2010 CLSI pubblicazione CLSI M5I-A “Method for antifungal disk diffusion susceptibility testing of nondermatophytes filamentous fungi; Approved Guideline"

2010 CLSI pubblicazione CLSI M5I-SI "Performance Standards for Antifungal Disk diffusion susceptibility testing of filamentous fungi; Informational supplement" 
dei test in vitro di sensibilità agli antifungini. Risale al 2008 la prima proposta di standardizzazione dei test di sensibilità in vitro da parte di EUCAST.

Pare opportuno segnalare, tuttavia, che proprio in ambito micologico vennero introdotte, già nel 1997 due nuove categorie interpretative dei risultati di MIC aggiuntesi a quelle ben note di Sensibile ("la terapia appropriata è quella effettuata alla dose raccomandata per la specie e per il tipo di infezione"), Intermedio ("i ceppi con MIC prossima a quella raggiungibile nel sangue per $\mathrm{i}$ quali la risposta è inferiore a quella dei sensibili o non ci sono dati per determinare la categoria come S o R") e Resistente ("i ceppi non sono inibiti alle concentrazioni raggiungibili con le dosi normali o nei casi in cui gli studi di terapia non hanno dimostrato efficacia clinica").

$\mathrm{Si}$ tratta delle categorie di: 1. Non Suscettibile (NS), con cui sono indicati i dati relativi ad antimicotici nuovi per i quali non sono stati isolati ancora ceppi resistenti e 2. Sensibile DoseDipendente (S-DD), da riservare ai casi in cui la MIC del farmaco si approssima a quella raggiungibile nel sangue e nei tessuti ma la cui risposta può essere inferiore a quella dei ceppi $\mathrm{S}$. Tale criterio - ipotizzato da Rex per qualsiasi specie di Candida che presentasse valori di MIC per fluconazolo compresi tra 16 e $32 \mathrm{mcg} / \mathrm{mL}$ sulla base della risposta clinica ottenuta a seguito di somministrazioni giornaliere alla posologia non inferiore a $100 \mathrm{mg}$ di farmaco. Tale criterio interpretativo fu poi esteso anche ad itraconazolo - costituisce il primo tentativo organico di correlare il dato in vitro all'outcome clinico.

\section{I criteri per la determinazione dei valori di breakpoint}

La disponibilità di nuove molecole antifungine ha comportato nuove possibilità terapeutiche: per questo il clinico "chiede lumi" al microbiologo per scegliere il più appropriato schema di terapia antifungina. D'altra parte, ciò ha determinato anche l'accresciuto interesse per il microbiologo clinico a testare in vitro gli antifungini: per questo il microbiologo "chiede lumi" per scegliere i metodi di laboratorio più efficaci per realizzare $\mathrm{i}$ test di chemiosensibilità. Così, la possibilità di rilevare resistenze microbiologiche e/o cliniche che possono giustificare insuccessi terapeutici fa sì che sia il clinico sia il microbiologo "chiedano lumi" per correlare i risultati ottenuti in vitro con la predittività dell'outcome in vivo.

La filosofia sottesa ai criteri EUCAST per la definizione dei valori di breakpoint risente di una prospettiva di grande rigore metodologico che, conseguente ai presupposti dell'Evidence Based
Medicine, contempera i dati di epidemiologia microbica con quelli di risultato clinico, sviluppando criteri che siano in grado non solo di predire il successo clinico ma anche di migliorare la sensibilità del metodo analitico di microdiluizione in brodo per consentire l'individuazione più efficace dell'emergenza di resistenza tra le differenti specie di Candida testando le molecole di comprovata efficacia terapeutica.

Per questo motivo EUCAST considera, in accordo con le linee guida che le società scientifiche cliniche propongono in tema di gestione farmacologica delle micosi da lieviti, le molecole da utilizzare anche per i saggi di sensibilità in vitro.

In tale prospettiva, EUCAST - nel documento dell'AFST - considera il fluconazolo come terapia appropriata in caso di:

- candidemia in pazienti neutropenici e non neutropenici

- candidosi disseminata cronica

- candidosi cutanea disseminata neonatale

- infezioni del tratto urinario, infezioni delle basse vie respiratorie, infezioni dell'apparato osteo-articolare (osteomielite e artrite), infezioni addominali (colecistite, pancreatite e peritonite), del cuore e dei vasi (endocardite, pericardite, flebite suppurativa, miocardite) meningite e endoftalmite causate da Candida spp.

- candidosi mucocutanea non genitale

- candidosi genitale

Inoltre considera, il fluconazolo come farmaco appropriato per la profilassi di:

- pazienti neutropenici particolarmente se colonizzati da Candida tropicalis

- riceventi di trapianto allogenico di midollo osseo

- pazienti ad alto rischio riceventi di trapianto di fegato

Infine, EUCAST considera il voriconazolo come terapia appropriata in caso di infezione da Candida causate da specie wild type (WT) di C. albicans, $C$. tropicalis e C. parapsilosis in corso di:

1. candidemia in pazienti non neutropenici

2. candidosi invasiva

3. candidosi esofagea

Di recente, EUCAST ha proposto tentative breakpoint validi per alcune specie di Candida relativamente ad amfotericina B, posaconazolo e anidulafungina.

Posaconazolo è un antifungino triazolico attivo nei confronti di Candida spp. (con un'attività in vitro non uniforme per le diverse specie), Cryptococcus spp., Aspergillus spp. e altri miceti filamentosi. Per quanto riguarda le infezioni da Candida, esso approvato come terapia di prima linea delle candidosi orofaringee nei pazienti 
gravi o immunocompromessi, per i quali si sospetta una scarsa risposta alla terapia antifungina topica.

Anidulafungina è un'echinocandina attivo contro Candida spp. È considerato come farmaco appropriato per la terapia delle candidosi invasive nei pazienti adulti non-neutropenici. L'attività in vitro di anidulafungina contro le specie di Candida non è uniforme. Candida parapsilosis e C. guilliermondii presentano una MIC più elevata dovuta alla presenza di una sostituzione naturale aminoacidica nell'hot spot del gene dell'enzima target.

Da ciò consegue che l'AFST di EUCAST abbia definito gli standard operativi per rilevare la sensibilità dei lieviti fermentanti ed abbia proposto valori di breakpoint per le infezioni sostenute solo da alcune specie di Candida e solo per alcuni tra gli antifungini disponibili.

Per le micosi causate da altre specie di lieviti, EUCAST non ha ancora reso disponibili i valori di breakpoint clinici. Per questo ribadisce l'approccio clinico che il microbiologo deve tenere: raccomandare al clinico di evitare terapie con farmaci che risultano inattivi in vitro e con molecole che presentano valori di MIC elevata, cioè al di sopra del limite superiore di MIC che caratterizza la popolazione WT: epidemiological cut-off (ECOFF), per le specie fungine causa di micosi. Al proposito, la distribuzione $W T$ per specie è definita come la distribuzione delle MIC dei ceppi che non hanno resistenza acquisita/mutazionale per l'antifungino in questione.

Per tale motivo l'AFST di EUCAST ha considerato, nell'elaborazione dei valori di ECOFF, cinque aspetti fondamentali:

1. il dosaggio più frequentemente utilizzato in ogni paese europeo;

2. la definizione, a livello di specie, di popolazione $W T$ per ogni microrganismo target e la determinazione dei valori di epidemiological cut-off;
3. le caratteristiche farmacocinetiche dei farmaci;

4. le caratteristiche farmacodinamiche degli antifungini, desunte da simulazioni Monte Carlo e

5. la correlazione delle MIC con l'outcome clinico dei pazienti trattati con ogni specifico antifungino.

Questa correlazione della MIC con l'outcome clinico veniva applicata in passato dal Clinical Laboratory Standard Institute (CLSI) statunitense applicando la regola "90-60": le infezioni da microrganismi suscettibili rispondono alla terapia in circa il $90 \%$ dei casi, mentre le infezioni da germi resistenti rispondono in circa il 60\%. Quindi, il breakpoint clinico di resistenza dovrebbe essere il valore di MIC associato a una risposta clinica di circa il $60 \%$. Tale approccio è soggetto a bias rilevanti come quello, fondamentale, rappresentato dall'osservatore. Per fluconazolo, Cuesta et al. (2010) introducendo strumenti significativi, in particolare il Classification and Regression Tree (CART), hanno permesso di realizzare una classificazione con migliore performance: questo sistema è quello utilizzato da EUCAST per sviluppare i breakpoint (9).

Qualora i dati disponibili fossero insufficienti (ad esempio, per la mancanza di informazioni conclusive sull'outcome clinico delle micosi sostenute da specie fungine caratterizzate da MIC elevate), l'AFST di EUCAST raccomanda di considerare, nell'impostazione di un adeguato schema di terapia, i valori di epidemiological cut-off epidemiologici, piuttosto che le concentrazioni breakpoint, fintantoché la letteratura scientifica non proponga dati definitivi di efficacia clinica desunti da studi controllati (17).

Deve essere, tuttavia, segnalato che EUCAST, a differenza di CLSI, utilizza la categoria terapeutica "I" invece di "SDD" per gli stipiti che non sono considerati né sensibili né resistenti. Parimenti,

Tabella 2. Cronistoria dei documenti EUCAST di standardizzazione dei test di sensibilità agli antifungini

\begin{tabular}{lll}
\hline Version* Date & Action \\
\hline 2.2 & $2010-03-22$ & $\begin{array}{l}\text { Voriconazole Candida krusei breakpoint changed from “--” to “IE” and Rationale } \\
\text { Document by the EUCAST Subcommittee on Antifungal Susceptibility Testing (EUCAST } \\
\text { AFST) updated. }\end{array}$ \\
\hline 2.1 & $2008-08-27$ & $\begin{array}{l}\text { Voriconazole Rationale Document by the EUCAST Subcommittee on Antifungal } \\
\text { Susceptibility Testing (EUCAST AFST) published on EUCAST website }\end{array}$ \\
\hline 2.0 & $2008-07-24$ & $\begin{array}{l}\text { Voriconazole breakpoints as determined by the EUCAST Subcommittee on Antifungal } \\
\text { Susceptibility Testing (EUCAST AFST) made publicly available }\end{array}$ \\
\hline 1.0 & $2007-07-10$ & $\begin{array}{l}\text { Fluconazole breakpoints as determined by the EUCAST Subcommittee on Antifungal } \\
\text { Susceptibility Testing (EUCAST AFST) made publicly available }\end{array}$ \\
\hline 1.0 & $2010-11-19$ & Amphotericin B. Rationale for the EUCAST clinical breakpoints \\
\hline 1.0 & $2010-11-19$ & Posaconazole. Rationale for the EUCAST clinical breakpoints. \\
\hline 1.0 & $2010-11-19$ & Anidulafungin. Rationale for the EUCAST clinical breakpoints \\
\hline & $2011-03$ & Consultation on clinical breakpoints for amphotericin B, posaconazole and anidulafungin \\
\hline
\end{tabular}


EUCAST in una nota indica che, quando il risultato è espresso con l'acronimo IE ("evidenza insufficiente"), la concentrazione breakpoint è denied: quindi è necessario che sul referto sia riportato il solo valore di MIC senza la traduzione in categoria terapeutica oppure che il laboratorio corredi l'esito con una nota informativa che espliciti l'attuale indisponibilità di criteri di breakpoint per questa specie fungina.

La Tabella 2 riporta la cronistoria dei documenti EUCAST in merito ai test di sensibilità agli antifungini.

\section{Le modalità tecniche di rilevazione della MIC} Il metodo di riferimento EUCAST è quello in brododiluizione.

Sono disponibili le modalità operative proposte da EUCAST per l'esecuzione dei test di sensibilità nei confronti dei lieviti fermentanti il glucosio (EUCAST Edef 7.1, 2008) (10). EUCAST ha deciso, inoltre, di sviluppare uno standard separato per i lieviti non fermentanti come Cryptococcus spp. o Trichosporon spp., a causa delle caratteristiche particolari, ma questo ancora non è stato approvato. Presupposto importante nell'approccio EUCAST per definire il valore dei cosiddetti breakpoint clinici è quello di evitare a tutti i costi di designare concentrazioni che dividano la distribuzione delle MIC dei ceppi $W T$ per ogni specie. Le principali differenze operative tra la metodologia proposta da EUCAST e quella di CLSI M27A3 (4) sono evidenziate nella Tabella 4.

Il test è eseguito utilizzando piastre microtiter con fondo piatto. Il metodo si basa nella preparazione di soluzioni degli antifungini che sono poi depositate - con la corrispondente concentrazione $(2 \mathrm{x}$ concentrazione finale) in volume di $100 \mu \mathrm{L} /$ - nei singoli pozzetti cui viene aggiunto l'inoculo fungino di $100 \mu \mathrm{L} /$ pozzetto.

Il terreno indicato è RPMI-1640 senza bicarbonato ma supplementato con glutamina, con un indicatore del $\mathrm{pH}$, e $2 \%$ di glucosio. Il buffer raccomandato è il 3-(N-morpholino)-propanesulphonic acid (MOPS) utilizzato ad una concentrazione finale di $0.165 \mathrm{~mol} / \mathrm{L}$ a $\mathrm{pH}$ 7.0.

L'inoculo viene preparato sospendendo cinque colonie, ottenute da una coltura di 24 ore in terreno di Sabouraud Destrosio Agar o Patata Destrosio Agar, in $5 \mathrm{~mL}$ di acqua distillata sterile e aggiustando poi la sospensione ad un McFarland $0.5\left(1-5 \times 10^{6} \mathrm{CFU} / \mathrm{mL}\right)$. La sospensione di lavoro si prepara diluendo 1:10 la sospensione standardizzata in acqua distillata sterile (inoculo finale: $0.5 \times 10^{5}$ a $2.5 \times 10^{5} \mathrm{CFU} / \mathrm{mL}$ ). È necessario allestire anche un pozzetto di controllo della crescita senza antimicotico.

Le piastre sono incubate per $24 \pm 2$ ore a $35 \pm 2{ }^{\circ} \mathrm{C}$ in atmosfera aerobia. Se la crescita è stentata (assorbanza $\leq 0.2$ ) dopo 24 ore - come può essere osservato con alcuni ceppi di C. parapsilosis e $C$. guilliermondii - è necessario reincubare per un massimo di altre 12-24 ore.

La lettura dell'assorbanza mediante lettore deve essere eseguita a $530 \mathrm{~nm}$, anche se possono essere usate altre lunghezze d'onda (405-450 nm), sottraendo la lettura del bianco ai valori dei pozzetti con antimicotici. La MIC dell'amfotericina B corrisponde al valore della concentrazione più bassa che determina un'inibizione della crescita $\geq 90 \%$ in rapporto al controllo senza antimicotico. Le MIC per 5-fluorocitosina ed azoli sono rappresentate dal valore della concentrazione più bassa che determina l'inibizione della crescita $\geq 50 \%$ in rapporto a quanto osservato nel pozzetto di controllo senza antimicotico.

La qualità dei risultati dei test deve essere monitorata utilizzando ceppi di controllo. Sono disponibili i valori di MIC per gli antifungini per i seguenti ceppi di controllo: C. krusei ATCC 6258, C. parapsilosis ATCC 22019, C. albicans F 8555 e C. krusei CL3403. Devono essere utilizzati almeno due ceppi di controllo, coltivati in terreno fresco non selettivo ogni qualvolta viene eseguito il test. I valori di MIC ottenuti devono essere compresi entro gli intervalli indicati in Tabella 3.

Per quanto riguarda la possibilità di definire il profilo di sensibilità con la metodica di disco-diffusione in agar, EUCAST non ha prodotto un documento di standardizzazione operativa. È disponibile però la tecnica descritta da CLSI, per la quale, tuttavia, i criteri interpretativi dei diametri di inibizione corrispondenti ai valori di breakpoint sono stati calcolati in rapporto a quelli stabiliti dallo stesso CLSI (M44-A2 e M44-S3) (5, 6). Nella metodica della disco-diffusione del CLSI (M44-A2 e M44S3) i diametri con i nuovi breakpoint per fluconazolo (disco con $25 \mathrm{mcg}$ ) per C. albicans, C. parapsilosis, e C. tropicalis si modificherebbero così: S, $\mathrm{MIC}, \leq 2 \mathrm{mcg} / \mathrm{mL}(\geq 17 \mathrm{~mm})$, SDD, MIC 4 $\mathrm{mcg} / \mathrm{mL}(14-16 \mathrm{~mm}), \mathrm{R}, \mathrm{MIC} \geq 8 \mathrm{mcg} / \mathrm{mL}(\leq 13$ $\mathrm{mm})$ e per $C$. glabrata, SDD $\leq 32 \mathrm{mcg} / \mathrm{mL},(\geq 15$ $\mathrm{mm}), \mathrm{R}, \geq 64 \mathrm{mcg} / \mathrm{mL}$ ( $\leq 14 \mathrm{~mm})(15)$.

\section{I criteri interpretativi per la definizione delle MIC secondo EUCAST}

Per quanto concerne specificatamente i criteri interpretativi utilizzati per la definizione delle $\mathrm{MIC}$, è fondamentale ricordare che i valori di breakpoint proposti da EUCAST per fluconazolo si riferiscono esclusivamente al caso di somministrazioni orali o intravenose, in vivo, di 400-800 $\mathrm{mg} /$ die. Analogamente, i valori di breakpoint di EUCAST per voriconazolo si riferiscono a poso- 
logie di $12 \mathrm{mg} / \mathrm{Kg}$ il primo giorno e poi 8 $\mathrm{mg} / \mathrm{Kg} /$ die, somministrate e.v., oppure di $800 \mathrm{mg}$ il primo giorno e poi di $400 \mathrm{mg} /$ die per pazienti di peso $>40 \mathrm{Kg}$ somministrate p.o. , oppure di 400 $\mathrm{mg}$ il primo giorno e poi di $200 \mathrm{mg} /$ die per soggetti di peso $<40 \mathrm{Kg}$, sempre p.o.

Del tutto analogamente, i valori di concentrazione breakpoint interpretativi per i lieviti fermentanti il glucosio sono stati stabiliti da EUCAST solo per fluconazolo e voriconazolo, molecole per le quali sono disponibili evidenze certe di efficacia clinica, nei confronti di C. albicans, C. parapsilosis e C. tropicalis. Candida krusei è sempre resistente al fluconazolo indipendentemente del risultato del test in vitro e, pertanto, il saggio in vitro non è affatto giustificato anche se è ammesso refertarne l'esito di resistenza esclusivamente sulla base di criteri epidemiologici. Non è stata ancora dimostrata, d'altra parte, un'evidenza sufficiente per i breakpoint clinici per C. glabrata (fluconazolo e voriconazolo) e C. krusei (voriconazolo).

D'altra parte, dal punto di vista interpretativo si segnala che il Sottocomitato per i test di sensibilità agli antifungini del CLSI ha recentemente modificato i breakpoint clinici per il fluconazolo per $C$. albicans, C. parapsilosis e C. tropicalis, armonizzandoli con quelli di EUCAST $(\mathrm{S}, \leq 2 \mathrm{mcg} / \mathrm{mL}$; $\mathrm{SDD}, 4 \mathrm{mcg} / \mathrm{mL}, \mathrm{R} \geq 8 \mathrm{mcg} / \mathrm{mL}$ ) mentre ha propo- sto modificare quelli di $C$. glabrata, eliminando la categoria $\mathrm{S}$ con i seguenti breakpoint: SDD, $\leq 32$ $\mathrm{mcg} / \mathrm{mL}, \mathrm{R} \geq 64 \mathrm{mcg} / \mathrm{mL}$. I breakpoint clinici adottati per fluconazolo per C. albicans, C. parapsilosis e C. tropicalis, potrebbero essere gli stessi anche per $C$. dubliniensis e C. lusitaniae, ma non per C. guilliermondii, che presenta un ECV a fluconazolo di $8 \mathrm{mcg} / \mathrm{mL}$ (95.9\%) (15).

Nonostante le differenze metodologiche tra i metodi EUCAST e CLSI di realizzazione operativa dei test di sensibilità, la valutazione comparativa in vitro degli azoli evidenzia che il valore della MIC per fluconazolo, qualora si utilizzino i breakpoint interpretativi EUCAST, presenta un'elevata concordanza quantitativa ( \pm 2 diluizioni): $95 \%$ e qualitativa: $95.8 \%$ (15). lasciando intravedere una possibile omologazione bidirezionale tra le due tecniche.

Per quanto attiene alle tre echinocandine, con entrambi i metodi di riferimento, EUCAST e CLSI, le MIC sono superiori nei ceppi con mutazioni fks che in quelli wild-type (WT), ma il range di quelli sensibili e di quelli con mutanti con frequenza si sovrappongono uno all'altro o sono separati solo di una o due diluizioni, risultando difficile l'identificazione corretta dei ceppi mutanti. L'aggiunta di $50 \mathrm{mg} / \mathrm{ml}$ di albumina di siero bovino al terreno proposto da EUCAST per-

Tabella 3. CQI lieviti fermentanti il glucosio con la metodica EUCAST: MIC accettabili i mcg/mL

\begin{tabular}{ccccc}
\hline Antifungino & $\begin{array}{c}\text { Candida krusei } \\
\text { ATCC 6258 }\end{array}$ & $\begin{array}{c}\text { Candida parapsilosis } \\
\text { ATCC 220I9 }\end{array}$ & $\begin{array}{c}\text { Candida albicans } \\
\text { F 8555 }\end{array}$ & $\begin{array}{c}\text { Candida krusei } \\
\text { CL3403 }\end{array}$ \\
\hline Amfotericina B & $0.12-1.0$ & $0.12-1.0$ & $0.06-0.5$ & $0.25-1.0$ \\
\hline Fluorocitosina & $1.0-4.0$ & $0.12-0.5$ & $0.06-0.25$ & $2.0-8.0$ \\
\hline Fluconazolo & $16.0-64.0$ & $0.5-2.0$ & $32.0-128.0$ & $16.0-64.0$ \\
\hline Itraconazolo & $0.03-0.12$ & $0.03-0.12$ & $0.25-1.0$ & $0.12-0.5$ \\
\hline Voriconazolo & $0.03-0.25$ & $0.015-0.06$ & $0.5-2.0$ & $0.12-0.5$ \\
\hline Posaconazolo & $0.015-0.06$ & $0.015-0.06$ & $0.12-0.5$ & $0.06-0.25$ \\
\hline Caspofungina & $\mathrm{ND}$ & $\mathrm{ND}$ & $\mathrm{ND}$ & $\mathrm{ND}$ \\
\hline Anidulafungina & $\mathrm{ND}$ & $\mathrm{ND}$ & $\mathrm{ND}$ & $\mathrm{ND}$ \\
\hline $\mathrm{ND}$ non disponibile & & & &
\end{tabular}

ND, non disponibile

Tabella 4. Principali differenze tra le metodiche CLSI e EUCAST per i test di sensibilità dei lieviti

\begin{tabular}{lcc}
\hline & CLSIM27A3 & EUCAST EDef 7.I \\
\hline Concentrazione di glucosio in RPMI I640 & $0.2 \%$ & $2 \%$ \\
\hline Forma del pozzetto della piastra Microtiter & Fondo ad “U” & Fondo piatto \\
\hline Dimensione dell'inoculo & $0.5-2.5 \times 10^{3}$ & $0.5-2.5 \times 10^{5}$ \\
\hline Tempo d'incubazione & 48 ore* & 24 ore \\
\hline Lettura & visiva & spettrofotometrica
\end{tabular}

Endpoint

Amfotericina B

Inibizione totale

Azoli ed echinocandine
Diminuzione prominente
La più bassa concentrazione capace d'inibire la crescita $\leq 90 \%$

La più bassa concentrazione capace d'inibire la crescita $\leq 50 \%$

*tranne che per le echinocandine, che si leggono alle 24 ore, e per il fluconazolo ove la lettura alle 24 ore è un optional. 
mette una migliore differenziazione tra i ceppi WT e quelli con mutanti di resistenza (2).

Per EUCAST nessun test è considerato perfetto per la valutazione in vitro delle echinocandine nei confronti dei lieviti, anche se le prove per anidulafungina identificano con buona affidabilità i mutanti $f k s$, qualora si utilizzi, per definire il breakpoint di sensibilità, il wild-type-upper-limit value (WT-UL) corrispondente a due doppie diluizioni sopra la concentrazione che individua il valore di $\mathrm{MIC}_{50}$.

Per quanto concerne la valutazione delle echinocandine, studi che comparano i risultati di saggi effettuati in accordo ai criteri proposti da EUCAST Edef 7.1 e da CLSI M27-A3 hanno dimostrato una buona correlazione (entro \pm 2 diluizioni) tra entrambi i metodi: la concordanza ottenuta per caspofungina: $89.5 \%$ e per micafungina: $99.2 \%$ suggerisce che è possibile l'armonizzazione tra entrambi gli approcci (14). I breakpoint CLSI per le echinocandine sono di $\leq 2 \mathrm{mg} / \mathrm{L}$, ma recentemente è apparso evidente che la maggior parte dei ceppi con mutazione $f k s 1$ e/o $f k s 2$ non necessariamente presentano valori di MIC superiori a questo breakpoint (1).

Il cut off epidemiologico per le echinocandine varia in funzione della specie e della molecola: per caspofungina varia da $0.12 \mathrm{mg} / \mathrm{L}$ (C. albicans, C. tropicalis e C. glabrata) a $4 \mathrm{mg} / \mathrm{L}(C$. guilliermondii); per micafungina da $0.03 \mathrm{mg} / \mathrm{L}$ (C. albicans e $C$. glabrata) a $4 \mathrm{mg} / \mathrm{L}(C$. guilliermondii $\mathrm{e}$ C. parapsilosis); per anidulafungina da $0.12 \mathrm{mg} / \mathrm{L}$ (C. albicans, C. tropicalis e C. krusei) a $16 \mathrm{mg} / \mathrm{L}$ (C. guilliermondii) (Pfaller maggio 2010).

Quindi, per alcune specie una concentrazione breakpoint con valore di MIC più basso, $0.25 \mathrm{o}$ $0.5 \mathrm{mg} / \mathrm{L}$, può risultare più sensibile per determinare i ceppi con mutazioni $f s k$, e pertanto non sensibili alle echinocandine.

I nuovi valori di breakpoint proposti dal CLSI per le tre echinocandine sono per C. albicans, C. tropicalis e C. krusei: Sensibile $\leq 0.25 \mathrm{mg} / \mathrm{L}$, Resistente $\geq 1 \mathrm{mg} / \mathrm{L}$; per $C$. parapsilosis: Sensibile $\leq 2 \mathrm{mg} / \mathrm{L}$, Resistente $\geq 8 \mathrm{mg} / \mathrm{L}$; e per
C. glabrata per Caspofungina e Anidulafungina Sensibile $\leq 0.12 \mathrm{mg} / \mathrm{L}$, Resistente $\geq 0.5 \mathrm{mg} / \mathrm{L}$;mentre per Micafungina Sensibile $\leq 0.06 \mathrm{mg} / \mathrm{L}$, Resistente $\geq 0.25 \mathrm{mg} / \mathrm{L}$ (16).

La Tabella 5 riporta i criteri di interpretazione secondo EUCAST.

\section{Le modalità operative per l'esecuzione dei test di sensibilità agli antifungini}

Poiché la metodica della brododiluizione è tecnica costosa, time consuming, che richiede esperienza da parte degli operatori e, per questo, è assolutamente poco praticata nei laboratori di diagnostica microbiologica, sono state sviluppate metodiche commerciali di più agevole applicabilità.

Non tutte queste tecniche si caratterizzano, tuttavia, per una buona correlazione con le procedure di riferimento: per questo si raccomanda che i laboratori di microbiologia utilizzino esclusivamente i metodi commerciali di cui siano disponibili elementi consolidati che ne garantiscano la comparabilità a quelli di riferimento (7).

Le tecniche di riferimento sono raccomandabili solo nei laboratori specializzati che eseguono studi di sensibilità per altre ragioni, come la validazione di nuove tecniche, la valutazione di nuove molecole o il saggio di specie fungine rare. Inoltre, le procedure di riferimento sono mandatarie per stabilire i breakpoint e negli studi di sorveglianza per determinare le resistenze.

In un recente studio, la correlazione tra i risultati per i lieviti della metodica EUCAST con i sistemi Vitek2, card AST-YS01 (bioMérieux), Etest (bioMérieux) e Sensititre YeastOne (Trek Diagnostic Systems) per amfotericina B, 5-fluorocitosina, fluconazolo e voriconazolo è risultata $>95 \%$ (entro \pm 2 diluizioni) con tutte le metodiche e per tutti i quattro farmaci antifungini (8).

In particolare, la concordanza dell'EUCAST con i tre sistemi commerciali era $>97 \%$ per il fluconazolo e $>97 \%$ con il Vitek 2 e $>95 \%$ con l'Etest e con il YeastOne per il voriconazolo. Very major errors (ceppo classificato come resistente in vitro con il metodo commerciale e sensibile con

Tabella 5. Breakpoint EUCAST per Candida Spp. $(12,13)$

\begin{tabular}{lccccc}
\hline \multirow{2}{*}{ Antifungino } & \multicolumn{5}{c}{ Breakpoint specie-correlati $(\mathrm{S} \leq / \mathrm{R}>$ ) } \\
\cline { 2 - 6 } & C. albicans & C. glabrata & C. krusei & C. parapsilosis & C. tropicalis \\
\hline Fluconazolo & $2 / 4$ & $\mathrm{IE}$ & $-(\mathrm{R})$ & $2 / 4$ & $2 / 4$ \\
\hline Voriconazolo & $0,125 / 0.125$ & $\mathrm{IE}$ & $\mathrm{IE}$ & $0.125 / 0.125$ & $0.125 / 0.125$ \\
\hline Amfotericina B* & $\mathrm{I} / \mathrm{I}$ & $\mathrm{I} / \mathrm{I}$ & $\mathrm{I} / \mathrm{I}$ & $\mathrm{I} / \mathrm{I}$ & $\mathrm{I} / \mathrm{I}$ \\
\hline Posaconazolo* & $0.06 / 0.06$ & $\mathrm{IE}$ & $\mathrm{IE}$ & $0.06 / 0.06$ & $0.06 / 0.06$ \\
\hline Anidulafungina* & $0.03 / 0.03$ & $0.06 / 0.06$ & $0.06 / 0.06$ & - & $0.06 / 0.06$ \\
\hline *Tentative breakpoint & \multicolumn{5}{c}{} \\
"IE": evidenza insufficiente per determinare i breakpoint \\
"-": non breakpoint perché questa specie non è un buon target per l'antimicotico
\end{tabular}


l'EUCAST) sono stati osservati per il fluconazolo, nel 2.7\% dei ceppi con il Vitek 2, e nel 2.3\% con l'Etest ed il YeastOne e corrispondevano a ceppi di lenta crescita (4 ceppi di C. albicans, 1 di C. gulliermondii e 3 C. neoformans).

\section{Le indicazioni all'esecuzione dei test di sensibi- lità agli antifungini}

Non esistono chiare evidenze che supportino la raccomandazione di eseguire i test di sensibilità agli antifungini nella pratica clinica.

Nonostante ciò, sono evidenziabili situazioni cliniche nelle quali gli studi di sensibilità in vitro possono garantire al clinico informazioni utili per il management del singolo caso.

I test di sensibilità devono senz'altro essere eseguiti di routine in caso di: 1 . fallimento terapeutico, 2. fungemie breakthrough, 3. nei pazienti che siano stati, in tempi precedenti, sottoposti a schemi di profilassi antifungina e 4. nei casi in cui il quadro di micosi profonda sia sostenuta da lieviti di specie poco comuni, con profilo di sensibilità non conosciuto.

Un'ultima indicazione all'esecuzione dei test di sensibilità sono le finalità epidemiologiche: diversi studi hanno dimostrato che la sorveglianza epidemiologica delle infezioni fungine aiuta a determinare la sensibilità delle differenti specie e la loro prevalenza, e che ciò permette la scelta della terapia più appropriata.

\section{Un problema aperto: il caso della candidosi vulvovaginale recidivante}

Tra le criticità emergenti che si osservano in clinica è il fallimento della terapia con fluconazolo in caso di vulvovaginite ricorrente (RVVC): in questi casi il clinico è indotto a chiedere al laboratorio l'esecuzione del test di sensibilità nei confronti dei lieviti isolati in coltura. I valori di breakpoint stabiliti da EUCAST per fluconazolo non sono, in realtà, applicabili ai casi di candidosi genitale. Già Sobel (2003) aveva osservato che la maggior parte $(84.9 \%)$ dei fallimenti terapeutici in caso di mucosite vaginale da $C$. albicans si osservavano nonostante l'elevato livello di sensibilità a fluconazolo $(\mathrm{MIC} \leq 1 \mathrm{mg} / \mathrm{L})$, presentato dai ceppi fungini isolati in coltura, nonostante la resistenza di C. albicans a fluconazolo nelle vaginiti complicate fosse evento raro e nonostante $\mathrm{i}$ ceppi di C. albicans con MIC elevata fossero manifestassero maggiore persistenza (19).

Si deve, peraltro, segnalare che, alla dose abituale di $150 \mathrm{mg}$ di fluconazolo somministrato per os, la concentrazione di triazolo raggiunta nelle secrezioni vaginali è di $2 \mu \mathrm{g} / \mathrm{mL}$. Ciò è dovuto alla presenza di acidi organici, in particolare di acetato, a livello vaginale, che esercita un effetto sinergico a fluconazolo. Tale sinergia è dovuta al fatto che, in vivo a livello della mucosa vaginale, fluconazolo non esercita più un effetto fungi statico ma fungicida. Così, l'attività fungicida nei confronti di C. albicans può essere determinata, in vivo, dall'attività dell'acetato che aumenta la permeabilità della membrana o diminuisce l'efflusso del fluconazolo. I ceppi di C. albicans resistenti nelle condizioni standard sono neutralizzati dall'associazione tra fluconazolo ed acetato, suggerendo che la terapia può essere potenzialmente efficace anche in presenza di ceppi di C. albicans con MIC elevate al fluconazolo. Lo stesso fenomeno si verifica anche con altre specie di Candida, ma certamente non nel caso di C. glabrata e di C. krusei. In anni recenti, tuttavia, Shahid e Sobel (2009) hanno registrato significative modificazioni epidemiologiche riguardanti la sensibilità al fluconazolo anche in C. albicans di isolamento vaginale: la prevalenza dei ceppi caratterizzati da MIC $\geq 1$ $\mu \mathrm{g} / \mathrm{mL} \mathrm{o} \geq 2 \mu \mathrm{g} / \mathrm{mL}$, si è triplicata nel'arco di un trentennio, passando dal 3\% (1986-1989) al 9\% (2005-2008). Le implicazioni di questo incremento dei valori di MIC sono, ad oggi, ancora poco chiare ma, considerati i valori di concentrazione raggiunti da fluconazolo a livello vaginale, una riduzione della sensibilità potrebbe avere rilevanza clinica (18).

Per questo motivo Shahid e Sobel suggeriscono l'adozione di un valore di concentrazione breakpoint alternativo, pari a $1 \mu \mathrm{g} / \mathrm{L}$, quando si testi in vitro il fluconazolo nei confronti dei ceppi di $C$. albicans di isolamento vaginale.

Nei casi in cui l'eziologia della vaginite fungina sia sostenuta da C. glabrata o da C. krusei, che per definizione presentano valori di MIC per fluconazolo superiori alla concentrazione raggiungibile a livello vaginale, la terapia con fluconazolo dà risultati clinici e micologici del tutto modesti tali da indurre ad adottare un approccio terapeutico alternativo, basato sull'utilizzo, in formulazioni topiche, di irrigazioni vaginali di acido borico, di 5-fluorocitosina o di amfotericina B.

L'esecuzione dei test di sensibilità non è, pertanto, mai indicata in caso di isolamento di C. albicans in forme sporadiche di vulvovaginite micotica.

$\mathrm{Al}$ contrario, essa può trovare indicazione in taluni, selezionati, casi di RVVC: non certo nelle forme da C. glabrata o da C. krusei, per le quali la scelta dello schema terapeutico deriva solo dalla corretta diagnosi eziologica e poggia sulla scelta di trattamenti topici, ma in caso di infezione da $C$. albicans refrattaria o breakthrough o caratterizzata da ricadute frequenti di terapia (frequent relapses of therapy), per verificare la possibilità che il fallimento terapeutico sia dovuto ad una diminuzione della sensibilità di C. albicans al fluconazolo. 


\section{Le raccomandazioni del Comitato di Studio per la Micologia (CoSM)}

Il Comitato di Studio per la Micologia (CoSM) dell'Associazione Microbiologi Clinici Italiani ritiene opportuno, in conclusione, procedere alle segnalazioni delle seguenti raccomandazioni per l'esecuzione, nella pratica diagnostica routinaria, dei test di sensibilità agli antifungini in corso di patologie sostenute da lieviti:

1. garantire l'identificazione di specie dei lieviti isolati in coltura: questa è l'azione fondamentale che il laboratorio deve effettuare per consentire, in ogni circostanza, la corretta impostazione dello schema terapeutico, indipendentemente dall'esecuzione dei test di sensibilità;

2. eseguire i test di sensibilità in vitro esclusivamente nel caso si verifichino le condizioni di seguito riportate:

A. - di natura clinica:

- micosi invasive

- micosi in soggetti immunocompromessi

- fungemie "breakthrough"

- fallimento terapeutico

- precedente profilassi antifungina

B. - di natura microbiologica:

- specie poco comuni

- specie la cui sensibilità non è prevedibile esclusivamente in base all'identificazione eziologica

3. eseguire, qualora necessario, i test di sensibilità utilizzando un metodo commerciale che consenta la determinazione delle MIC: Sensititre YeastOne, Etest o Vitek2, validato nei confronti dei metodi di riferimento EUCAST. In alternativa, laddove sia possibile, effettuare i saggi in accordo con le modalità operative standardizzate previste dal documento EUCAST. Nel caso non siano disponibili presso il laboratorio tali sistemi diagnostici, è opportuno riferire lo stipite fungino ad un centro di referenza;

4. selezionare accuratamente i breakpoint adeguati ed interpretare $\mathrm{i}$ risultati in accordo con $\mathrm{i}$ documenti EUCAST;

5. riferire i risultati riportando sul referto, oltre ai valori di MIC, anche le categorie terapeutiche S-I-R per i binomi molecola-lievito per i quali EUCAST dispone di criteri interpretativi. In caso contrario, per i binomi molecola-lievito per i quali EUCAST non fornisce la traduzione dei risultati in categorie terapeutiche, riportare solo il valore della MIC, segnalando, in una nota di commento che: "il test di sensibilità agli antifungini è stato interpretato in accordo con i criteri dell'European Committee on Antimicrobial Susceptibility Testing (EUCAST). I risultati dei test in vitro devono essere ritenuti affidabili solo nel caso in cui la posologia del farmaco utilizzata per la terapia corrisponde a dosaggi "pieni". Poiché per la specie fungina testata non sono ancora disponibili criteri di breakpoint che consentano una consolidata correlazione tra il valore di MIC e l'outcome clinico, i risultati devono essere valutati alla luce del quadro clinico da uno specialista in terapia anti-infettiva".

\section{BIBLIOGRAFIA}

1. Arendrup MC, Garcia-Effron G, Lass-Flörl C, et al. Echinocandin susceptibility testing of Candida species: comparison of EUCAST EDef 7.1, CLSI M27-A3, Etest, Disk diffusion, and Agar dilution method with RPMI and IsoSensitest media. Antimicrob Agents Chemother, 2010; 54: 426-39.

2. Arendrup MC, Rodriguez-Tudela J-L, Park S, et al. Echinocandin Susceptibility Testing of Candida spp. Using EUCAST EDef 7.1 and CLSI M27-A3 Standard Procedures: analysis of the influenze of bovine serum albumin supplementation, storage time, and drug lots. Antimicrob Agents Chemother, 2011; 55: $1580-7$.

3. Clinical and Laboratory Standards Institute. Method for Broth Dilution Antifungal Susceptibility Testing of Filamentous Fungi; Approved Standard-Second Edition. M38-A2, 2008; 28 (16): 1-35.

4. Clinical and Laboratory Standards Institute. Reference Method for Broth Dilution Antifungal Susceptibility Testing of Yeasts; Approved Standard-Third Edition. M27-A3, 2008; 28 (14): 1-25.

5. Clinical and Laboratory Standards Institute. Reference Method for Antifungal Disk Diffusion Susceptibility Testing of Yeast; Approved Standard. M44-A2, Clinical and Laboratory Standards Institute; Wayne, PA, 2008.

6. Clinical and Laboratory Standards Institute. Zone Diameter Interpretative Standards, Corresponding Minimal Inibitory Concentration. (MIC) Interpretative Breakpoints and Quality Control Limits for Antifungal Disk Diffusion Susceptibility Testing of Yeast: Informational Supplement, M44-S3. Clinical and Laboratory Standards Institute; Wayne, PA, 2009

7. Cuenca-Estrella M, Bassetti M, Lass-Flörl C, Ráčil Z. Richardson M, Rogers TR. Detection and investigation of invasive mould disease. $J$ Antimicorb Chemother, 2011; 66 Suppl 1: 115-24.

8. Cuenca-Estrella M, Gomez-Lopez A, AlastrueyIzquierdo A, et al. Comparison of the Vitek 2 Antifungal Susceptibility System with the Clinical and Laboratory Standards Institute (CLSI) and European Committee on Antimicrobial Susceptibility Testing (EUCAST) broth microdilution reference methods and with the Sensititre YeastOne and Etest techniques for in vitro detection of antifuangal resistance in yeast isolates. $J$ Clin Microbiol, 2010; 48: 1782-6.

9. Cuesta I, Bielza C, Cuenca-Estrella M, Larranaga P, Rodriguez-Tudela JL. Evaluation by data mining tecniches of fluconazole breakpoints established by the Clinical and Laboratory Standards Institute (CLSI) and comparison with those of the European Committee on Antimicrobial Susceptibility Testing (EUCAST). Antimicrob Agents Chemother, 2010; 54: 1541-6.

10. EUCAST Definitive Document E.Def 7.1: method for the determination of broth dilution MICs of antifungal 
agents for fermentative yeasts. Clin Microbiol Infect 2008; 14: 398-405.

11. EUCAST definitive document E.DEF 9.1: Method for the determination of broth dilution minimum inhibitory concentrations of antifungal agent for conidia forming moulds. Subcommittee on Antifungal Susceptibility Testing (AFST) of the ESCMID European Committee for Antimicrobial Susceptibility testing (EUCAST) http://www.eucast.org.

12. EUCAST Technical Note on fluconazole. Clin Microbiol Infect 2008; 14: 193-5.

13. EUCAST Technical Note on voriconazole. Clin Microbiol Infect 2008; 14: 985-7.

14. Pfaller MA, Castanheira M, Diejkema DJ, Messer SA, Moet GJ, Jones RN. Comparison of European Committee on Antimicrobial Susceptibility Testing (EUCAST) and Etest methods with the CLSI broth microdilution method for echinocandin susceptibility testing of Candida species. J Clin Microbiol, 2010; 48: 1592-9.

15. Pfaller MA, Andes D, Diekema DJ, Espinel-Ingroff A, Sheehan D. The CLSI Subcommittee for Antifungal Susceptibility Testing. Wild-type MIC distributions, epidemiological cutoff values and species-specific clinical breakpoints for fluconazole and Candida: Time to harmonization of CLSI and EUCAST broth microdilution methods. Drug Resistance Updates 2010; 13: 180-95.

16. Pfaller MA, Diekema DJ, Andes D, et al. The LSI Subcommittee for Antifungal Testing Clinical breakpoints for the echinocandins and Candida revisited: Integration of molecular, clinical, and microbiological data to arrive at species-specific interpretive criteria Drug Resistance Updates, 2011; 14: 164-76.

17. Rodriguez-Tudela JL, Arendrup MC, Cuenca-Estrella M, Donnelly JP, Lass-Flörl C. EUCAST breakpoints for antifungals. Drug News Perspect, 2010; 23: 93-7.

18. Shahid Z, Sobel JD. Reduced fluconazole susceptibility of Candida albicans isolates in women with recurrent vulvovaginal candidiasis: effects of long-term fluconazole therapy. Diagnostic Microbiol Infect Dis, 2009; 64: 354-6.

19. Sobel JD, Zervos M, Reed BD, et al. Fluconazole susceptibility of vaginal isolates obtained from women with complicated Candida vaginitis: clinical implications. Antimicrob Agents Chemother. 2003; 47: 34-8. 